\title{
Culturversuche mit isolierten Pflanzenzellen
}

von

G. Haberlandt, c. M. k. Akad.

(Mit 1 Tafel.)

(Vorgelegt in der Sitzung am 6. Februar 1902.)

I.

Es sind meines Wissens bisher noch keine planmäßig angelegten Versuche gemacht worden, isolierte vegetative Zellen von höher entwickelten Pflanzen in geeigneten Nährlösungen $\mathrm{zu}$ cultivieren. Und doch müssten die Ergebnisse solcher Culturversuche manches interessante Streiflicht auf die Eigenschaften und Fähigkeiten werfen, die die Zelle als Elementarorganismus in sich birgt, sie müssten Aufschlüsse bringen über die Wechselbeziehungen und gegenseitigen Beeinflussungen, denen die Zellen innerhalb des vielzelligen Gesammtorganismus ausgesetzt sind.

Ich habe bereits im Jahre 1898 eine Anzahl solcher Culturversuche mit künstlich isolierten Pflanzenzellen durchgeführt. Es war ursprünglich meine Absicht, diese Versuche in größerem Maßstabe fortzusetzen und die Zellen der verschiedensten Gewebearten auf ihr Verhalten bei der Cultur in verschiedenartigen Nährlösungen zu prüfen. Andere Arbeiten verhinderten die Ausführung dieses Planes, und da mich meine sinnesphysiologischen Untersuchungen wohl noch längere Zeit hindurch in Anspruch nehmen werden, so sei es mir gestattet, die Ergebnisse jener Versuche im Nachstehenden kurz mitzutheilen. 
$\mathrm{Zu}$ den Versuchen konnten natürlich nur solche Pflanzentheile verwendet werden, deren Zellen in lockerem gegenseitigen Verbande stehen, so dass sie auf mechanischem Wege leicht zu isolieren waren. Da ich zunächst grüne Assimilationszellen cultivieren wollte, so handelte es sich vor allem um die Auffindung entsprechend gebauter Laubblätter. Als sehr geeignet erwiesen sich in dieser Hinsicht die Hochblätter von Lamium purpureum, die typisches Palissaden- und Schwammgewebe besitzen. Kleine Blattfragmente wurden auf einem Objectträger in einigen Tropfen der Nährlösung mit zwei Nadeln so lange zerzupft, bis die Betrachtung mit einem schwachen Objectivsysteme das Vorhandensein zahlreicher isolierter Palissaden- und Schwammparenchymzellen ergab. Die Culturen wurden anfänglich im hängenden Tropfen vorgenommen, später in kleinen Glasschälchen mit Deckeln, die ungefähr $10 \mathrm{~cm}^{3}$ der Nährlösung enthielten. Die Übertragung in die Nährlösung erfolgte mittels einer fein ausgezogenen Glaspipette. Dieselbe diente auch dazu, um von Zeit zu Zeit einige Zellen aus dem Schälchen herauszufischen und behufs der mikroskopischen Untersuchung auf den Objectträger zu bringen. Die Culturschälchen standen auf einem Tische vor einem Nordwestfenster des botanischen Institutes. Sie waren hier gut beleuchtet, vor directer Insolation aber geschützt. Auch im Dunkeln wurden einige Versuche ausgeführt. Die Temperatur betrug entsprechend der Jahreszeit (April, Mai, Juni, September, October, November) 18 bis $24^{\circ} \mathrm{C}$.

Natürlich wurden verschiedene Maßregeln getroffen, um die Culturen möglichst bakterien- und pilzfrei zu erhalten, wenngleich sich eine vollständige Sterilisierung in dieser Hinsicht als kaum durchführbar und auch nicht nothwendig erwies. Die Objectträger, Deckgläschen, Stahlnadeln, Saugpipetten und Glasschälchen wurden vor dem Gebrauche stets mehrmals durch die Flamme eines Bunsen-Brenners gezogen, respective ausgeglüht, die Nährlösungen vorher aufgekocht. Die zu zerzupfenden Blattheile wurden mit sterilisiertem Wasser sorgfältig abgespült. So gelang es, wenigstens eine Anzahl von Culturen hinlänglich rein zu erhalten. Übrigens ist zu bemerken, dass die cultivierten Pflanzenzellen im allgemeinen durch das 
Vorhandensein nicht zu zahlreicher Bakterien in den Culturlösungen nur wenig in ihrem Gedeihen beeinträchtigt wurden. Als Nährlösungen dienten: Wasserleitungswasser, Knop'sche Nährstofflösung, ${ }^{1} 1$ - bis 5 procentige Rohrzuckerlösungen, Knop'sche Nährstofflösung mit Zusatz von Rohrzucker, Traubenzucker, Glycerin, Asparagin und Pepton in wechselnden Combinationen und Concentrationen.

II.

Bevor ich nun die Ergebnisse der mit den Assimilationszellen der Hochblätter von Lamium purpureum durchgeführten Culturversuche übersichtlich zusammenstelle und erörtere, möchte ich als Beispiel den Verlauf eines einzelnen Culturversuches nach den Angaben meines Tagebuches mittheilen.

Schälchencultur. Knop'sche Nährstofflösung. Beginn a $m$ 21. April. Durchschnittliche Länge der Palissadenzellen $50 \mu$, Breite $27 \mu$. Die Chlorophyllkörner stärkefrei. 22. April. Zellen unverändert. Die Engelmann'sche Bakterienmethode ergibt, dass die isolierten Zellen kräftig assimilieren.

23. A pri1. Zellen unverändert. In verschiedenen Zellen lassen sich in den Chlorophyllkörnern mit Jodwasser mehrere winzige Stärkekörnchen nachweisen.

24. A pril. Fast alle Zellen sind noch am Leben. Viele darunter sind beträchtlich gewachsen. Die Palissadenzellen wachsen relativ mehr in die Breite als in die Länge, zeigen das Bestreben, sich abzurunden. Oft nehmen sie eine birnenförmige Gestalt an. Die Chlorophyllkörner werden kleiner, sie sind gleichmäßig vertheilt oder an einem Zellende dicht nebeneinandergelagert.

28. April. Fast alle Zellen sind noch am Leben. Ihr Wachsthum ist noch weiter fortgeschritten. Einzelne Palissaden-

1 In der von Sachs (Vorlesungen über Pflanzenphysiologie, II. Aufl., S. 266) angegebenen Zusammensetzung: Auf $1000 \mathrm{~cm}^{3}$ Wasser $1 \mathrm{~g}$ salpetersaures Kali, $0.5 \mathrm{~g}$ Gips, $0.5 \mathrm{~g}$ schwefelsaure Magnesia, $0.5 \mathrm{~g}$ phosphorsaurer Kalk, Spuren von schwefelsaurem Eisenoxydul. 
zellen sind jetzt $70 \mu$ lang und $50 \mu$ breit. Die Chlorophyllkörner sind noch kleiner geworden und zeigen einen Stich ins Gelbliche. Mittels der Bakterienmethode lässt sich noch Assimilation nachweisen, doch ist dieselbe weniger kräftig.

30. A pril. Die Mehrzahl der Zellen lebt noch. Die Zellwände sind etwas dicker geworden. Normale Wanddicke $0 \cdot 7 \mu$, gegenwärtige Wanddicke 1 bis $1 \cdot 4 \mu$. Während die Membranen normaler Zellen sich mit Chlorzinkjod nur langsam und nicht intensiv blau färben, tritt diese Färbung bei den cultivierten Zellen rasch ein und wird auch intensiver. Der Durchmesser der gelblich gewordenen Chlorophyllkörner ist bis auf die Hälfte kleiner geworden: zu Beginn des Versuches 6 bis $8 \mu$, jetzt $3 \cdot 2$ bis $3 \cdot 8 \mu$. Die Chlorophyllkörner enthalten aber noch winzige Stärkekörner.

2. Mai. Alle Zellen todt. Der sehr dünne plasmatische Wandbeleg hat sich unter Faltenbildung von der Zellwand ein wenig abgehoben. Chlorophyllkörner noch kleiner, ganz blass, theilweise desorganisiert.

Die wichtigsten Versuchsergebnisse möchte ich in folgende Punkte zusammenfassen:

1. Bei der Cultur in diffusem Tageslichte lassen sich die Assimilationszellen viele Tage lang am Leben erhalten. In Knop'scher Nährlösung, also bei bloßer Ernährung mit anorganischen Stoffen, blieben die Zellen zuweilen drei Wochen lang am Leien (z. B. in einer Schälchencultur vom 2. bis 24. Mai). Noch länger lebten sie in Nährlösungen, die überdies $1 \%$ Rohrzucker enthielten. Hier waren auch nach einem Monate noch einzelne Zellen am Leben. Im Dunkeln gehen die Zellen weit rascher zugrunde, in Knop'scher Nährlösung schon nach 4 bis 6 Tagen, bei Zusatz von $1 \%$ Rohrzucker einige Tage später; noch länger blieben sie in 5 procentiger Rohrzuckerlösung am Leben.

2. Die Chlorophyllkörner assimilieren wenigstens in der ersten Zeit ganz kräftig. Festgestellt wurde dies mittels der Engelmann'schen Bakterienmethode und durch den Nachweis 
von Stärkekörnern in den zu Beginn des Versuches stärkefreien Chloroplasten.

Merkwürdig ist das verschiedene Verhalten der Chlorophyllkörner in Knop'scher Nährstofflösung und in 1- bis 5 procentigen Rohrzuckerlösungen. In ersterer werden sie allmählich kleiner, nehmen bald einen Stich ins Gelbliche an, werden dann blasser und können sich schließlich in ganz zart contourierte kleine Leukoplasten umwandeln. In Zuckerlösungen werden sie bei geringerer Concentration der Lösung (1\%) zwar auch kleiner, behalten aber ihre grüne Farbe; bei größerer Concentration (3 bis $5 \%$ ) nehmen sie an Größe nicht ab und erscheinen bis zum Tode der Zelle noch intensiv grün gefärbt, oft sogar noch intensiver als zu Beginn des Versuches. Es ist dabei gleichgiltig, ob die Zellen belichtet oder verdunkelt werden.

Wie ist nun dieses verschiedene Verhalten der Chlorophyllkörner zu erklären? Am nächsten liegt die Annahme, dass es sich um eine Ernährungsfrage handelt, dass die Chlorophyllkörner isolierter Zellen, wenn sie bloß auf ihre eigene Assimilationsthätigkeit angewiesen sind, sich nicht intact erhalten können, sondern allmählich verkümmern müssen: sie geben ihre Assimilationsproducte so vollständig an die übrigen Zellorgane $a b$, die dabei oft starkes Wachsthum zeigen, dass für die Erhaltung ihrer eigenen Integrität zu wenig übrig bleibt. Man muss dabei annehmen, dass durch die Wiederaufnahme des Wachsthums der Zelle das normale Verhältnis zwischen den Chlorophyllkörnern und den nicht grünen Zellorganen gestört wird und nun ein Verhältnis eintritt, wie es zwischen Wirt und Schmarotzer besteht; dabei würde es sich um jene einfachste Form des Parasitismus handeln, bei welcher der Parasit den Wirt durch Entzug von plastischen Baustoffen schädigt und schließlich zum Absterben bringt. Wenn man aber die Chlorophyllkörner durch Zufuhr von Zucker in ihrer Ernährungsthätigkeit unterstützt, respective entlastet, so können sie sich intact erhalten. Dass diese Erklärung richtig ist, geht auch aus den unten zu erwähnenden Culturversuchen mit den Assimilationszellen von Eichhormia crassipes hervor, in denen die zu Beginn des Versuches stärkefreien Chlorophyllkörner im 
Dunkeln rasch verkümmerten, während die stärkehältigen bis zum Verbrauche der Stärkekörner schön grün blieben.

Um die Chlorophyllkörner der Assimilationszellen von L. purpureum intact $\mathrm{zu}$ erhalten, genügte die Zufuhr von Zucker. Stickstoffhältige organische Substanzen waren dazu nicht erforderlich. In einer Nährlösung, die 3\% Rohrzucker und $1 \%$ Asparagin enthielt, blieben die Chlorophyllkörner zwar bis zuletzt lebhaft grün, doch zeigten sie weder Wachsthum noch Vermehrung. Auffallend war bloß ihre Neigung, sich abzuflachen und sich seitlich aneinander zu legen, namentlich in den Armenden der Schwammparenchymzellen, wo anscheinend einheitliche, große, muldenförmige Chloroplasten vorhanden waren. Die genauere Beobachtung ergab aber doch das Vorhandensein zarter Grenzlinien zwischen den einzelnen Chloroplasten.

Sehr eigenthümliche Gestaltsveränderungen, anscheinend mit Wachsthum verbunden, beobachtete ich an Chlorophyllkörnern einer fünftägigen Dunkelcultur in 5 procentiger Rohrzuckerlösung (Fig. 6). Die lebhaft grüngefärbten Chloroplasten waren mit tiefen Einkerbungen und Buchten versehen, so dass sie eine hufeisenförmige oder unregelmäßig gelappte Gestalt erhielten. Bisweilen waren die Lappen umgeschlagen. Diese Bildungsabweichungen erinnern lebhaft an jene eigenthümlichen Formen von Chloroplasten, wie ich sie bei verschiedenen Selaginellen (S. Martensii, Blattbasis; S. Kraussiana) beobachtet habe. ${ }^{1}$

3. Mit der fortdauernden Assimilationsthätigkeit war auch meist ein mehr oder minder ausgiebiges Wachsthum der isolierten Assimilationszellen verbunden. Sowohl die Palissaden-, wie die Schwammparenchymzellen zeigten ein solches. Die durchschnittliche Länge der normalen Palissadenzellen zu Beginn der Versuche betrug $50 \mu$, ihre Breite $27 \mu$. Nach erfolgtem Wachsthume waren sie im Maximum $108 \mu$ lang und $62 \mu$ breit (Fig. 1, 2). Daraus berechnet sich unter der Annahme einer cylindrischen Gestalt der Palissadenzellen eine Volumzunahme um das 11 fache des ursprünglichen Volums. Weniger

1 G. Haberlandt, Die Chlorophyllkörper der Selaginellen. Flora, 1888. 
stark, aber immerhin sehr ansehnlich war das Wachsthum der Schwammparenchymzellen. Sie vergrößertèn ihren Durchmesser von 38 bis $40 \mu$ auf 50 bis $68 \mu$. Die Palissadenzellen zeigten im allgemeinen ein stärkeres Breiten-, als Längenwachsthum; die Tendenz, sich abzurunden und der Kugelform zu nähern, trat deutlich zutage. Dass die Volumvergrößerung wirklich auf Wachsthum und nicht etwa bloß auf einer enormen elastischen Dehnung der Zellenmembran beruhte, gieng aus plasmolytischen Versuchen und aus den weiter unten zu besprechenden Wachsthumserscheinungen der Zellhaut ganz klar hervor.

Das Wachsthum der Assimilationszellen wurde durch Zusatz von Rohrzucker und Asparagin zur Nährlösung nicht begünstigt. Das ausgiebigste Wachsthum trat vielmehr in Knop'scher Nährstofflösung ein.

Aus den vorstehenden Angaben geht also hervor, dass isolierte Assimilationszellen das im normalen Entwickelungsgange abgeschlossene Wachsthum bei der Cultur in Nährlösungen in sehr ausgiebiger Weise wieder aufnehmen können. Was veranlasst sie dazu? Nur wenig wäre gewonnen, wenn man dieses erneute Wachsthum als eine Reaction auf den durch die Isolierung gesetzten Wundreiz (traumatische Reizung) betrachten würde. Denn diese Bezeichnung ist ein Sammelname für sehr verschiedene Einzelvorgänge, von denen jeder für sich als Reiz wirken kann. Die mechanische Zerrung der Protoplasten bei der Verletzung, die Zerreissung der Plasmaverbindungen, die Bloßlegung von Zellwänden, die früher an andere Zellen grenzten und die dadurch bedingte Steigerung der Transpiration, respective Erleichterung der Wasseraufnahme, die Unterbrechung des Stoffverkehres mit den Nachbarzellen, der Wegfall mechanischer und sonstiger Beeinflussungen seitens der benachbarten Gewebe und Organe, die Aufnahme von Zersetzungsproducten der bei der Verletzung zerstörten Zellkörper seitens der an die Wundfläche angrenzenden unverletzt gebliebenen Zellen: all dies und noch manche andere Änderung im bisherigen Gleichgewichtszustande macht in seiner Gesammtheit den Wundreiz aus. Wenn man daher eine bestimmte Reaction, die sich nach einem 
traumatischen Einflusse einstellt, als durch den Wundreiz bedingt ansieht, so ist dies im Grunde genommen nur eine Umschreibung der Thatsache und keine wissenschaftliche Einsicht.

Auch im vorliegenden Falle ist also eine präcisere Fragestellung nothwendig und im Nachstehenden sollen einige Möglichkeiten, die für die Erklärung des Wachsthums isolierter Assimilationszellen in Betracht kommen können, in Kürze discutiert werden.

Fangen die Zellen, wie man zunächst meinen möchte, nur deshalb wieder zu wachsen an, weil sie die plastischen Baustoffe, die sie producieren, nicht abgeben können? Dass dies nicht der alleinige oder ausschlaggebende Grund für den Wiederbeginn des Wachsthums sein kann, lehrt jedes Laubblatt, dessen Assimilationszellen im Laufe eines sonnigen Sommertages weit mehr Assimilationsproducte erzeugen, als sie in derselben Zeit abführen können, die aber trotzdem den Überschuss nicht zu eigenem Wachsthum verwenden, sondern provisorisch aufspeichern, bis im Laufe der Nacht die allmähliche Auswanderung erfolgt. Ebenso könnten ja auch die isolierten Assimilationszellen ihre Producte in sich aufspeichern, ohne sie zum Wachsthum zu verwenden.

Ein anderer Grund für den Wiederbeginn des Wachsthums könnte in der Beseitigung der mechanischen Wachsthumshindernisse gefunden werden, denen die im Gewebeverbande befindlichen Zellen ausgesetzt sind. In einem sehr locker gebauten Palissaden- und Schwammparenchym könnten aber diese Zellen reichlich in die lufterfüllten Intercellularen hineinwuchern, wenn sie überhaupt das Bestreben hätten, weiterzuwachsen.

Mit größerem Rechte ließe sich für den Wiederbeginn des Wachsthums der Reiz verantwortlich machen, den das veränderte Medium auf die Zellen ausübt. Im Blatte sind sie theilweise von Luft umgeben, in den Culturen ringsum von der Nährlösung. Die gesteigerte Wasseraufnahme könnte $z u$ erneutem Wachsthume führen. Ich halte aber eine solche directe Beeinflussung seitens des umgebenden Mediums für wenig wahrscheinlich. Als ich vor Jahren im botanischen Garten zu 
Buitenzorg die normalen Hydathoden des Laubblattes von Conocephalus ovatus Tréc. vergiftete ${ }^{1}$ und so eine täglich wiederkehrende Injection der Intercellularen mit Wasser herbeiführte, da zeigten die rings von der Flüssigkeit umspülten Palissaden- und Schwammparenchymzellen - von gewissen Stellen abgesehen - keine Veränderungen. Nicht die gesteigerte Wasserzufuhr als solche, sondern das Bedürfnis des Gesammtblattes nach Ausscheidung der überschüssigen Wassermengen führte zur localen Bildung zahlreicher Ersatzhydathoden. Die Leitparenchym- und Palissadenzellen, welche nunmehr an gewissen Stellen starkes Wachsthum zeigten, erhielten nicht mehr Wasser zugeführt, als die anderen Leitparenchym- und Palissadenzellen, die kein erneutes Wachsthum zeigten.

Auch die Möglichkeit, dass vielleicht die Aufnahme von Zersetzungsproducten der bei der Isolierung zerrissenen und getödteten Protoplasten seitens der isolierten intacten Zellen den Wiederbeginn des Wachsthums herbeiführe, muss zurückgewiesen werden. Denn wenn auch häufig bei der Isolierung einzelne todte Zellen oder Zellfragmente an den intacten Zellen hängen blieben, so war doch eine hinreichende Anzahl von Zellen vollständig isoliert. Dieselben zeigten das gleiche ausgiebige Wachsthum wie jene.

In der umgebenden Nährlösung konnten aber die fraglichen Zersetzungsproducte bei dem im Verhältnisse zum Schälcheninhalte äußerst geringen Volumen der eingeführten Zellen nur in so minimalen Mengen vorhanden sein, dass eine Reizwirkung ihrerseits wohl ausgeschlossen war.

Am wahrscheinlichsten ist wohl, dass die Wiederaufnahme des Wachsthums der Assimilationszellen nach ihrer Isolierung überhaupt nicht die Wirkung eines neu hinzutretenden Reizes ist, sondern dass die Zellen ihr unterbrochenes Wachsthum weiter fortsetzen, weil der seitens der Gesammtpflanze ausgehende Hemmungsreiz, der die Assimilationszellen des Blattes zwingt, in einem gewissen Stadium ihr

1 Vergl. G. Haberlandt, Über experimentelle Hervorrufung eines neuen Organes bei Conocephalus ovatus Tréc. Festschrift für Schwendener, 1899. 
Wachsthum einzustellen, nach der Isolierung der Zellen wegfällt. Wir wissen ja, dass im Organismus auf selbstregulatorische Weise verschiedenartige Vorgänge und Thätigkeiten bald angeregt, bald unterdrückt werden, ${ }^{1}$ wie es den Bedürfnissen des Gesammtorganismus entspricht. Im Interesse der möglichsten Leistungsfähigkeit eines Laubblattes liegt es offenbar, dass die Assimilationszellen eine gewisse Größe nicht überschreiten, denn die jeweilige Zellgröße einer bestimmten Gewebeart ist im Hinblick auf ihre physiologische Function ganz sicher ebenso eine zweckmäßige Eigenschaft, wie ihre Gestalt und ihre sonstigen morphologischen Merkmale. Ist diese Größe im ontogenetischen Entwickelungsgange erreicht, dann wird das weitere Wachsthum der Zelle sistiert, nicht weil die Zellen ihrer potentiellen Befähigung nach nicht weiter wachsen können, sondern weil vom Gesammtorganismus oder bestimmten Theilen desselben ein Reiz ausgeht - mag es nun eine rein dynamische oder stoffliche Beeinflussung sein durch welchen das Wachsthum zum Stillstande gelangt. Die isolierte Zelle kann dann das unterbrochene Wachsthum wieder aufnehmen.

Auch im Gesammtoryanismus mögen zuweilen, wenn er infolge von Störungen im Betriebe der Regulationsvorgänge die Herrschaft über einzelne Zellen oder ganze Zellcomplexe verliert, durch Wiederaufnahme des Wachsthums derselben »pathologische Hypertrophien « sich einstellen, die manche Ähnlichkeit mit jenen haben können, die durch directe Reizung, z. B. seitens verschiedener Parasiten, entstehen.

4. Die Zellmembranen der isolierten Assimilationszellen zeigen nicht bloß Flächen-, sondern auch Dickenwachsthum. In der oben mitgetheilten Cultur (S. 71) wuchs die Wanddicke in 9 Tagen von $0.7 \mu$ auf 1 bis $1.4 \mu$ heran. Abgesehen von dieser allgemeinen Dickenzunahme, die aber selten so auffällig war, stellten sich häufig auch locale Membranverdickungen ein. Die Palissadenzellen verdickten zuweilen ihre Querwände (Fig. 3), wobei die Verdickung in der Mitte am stärksten war und gegen den Rand

1 Vergl. Pieffer, Pflanzenphysiologie II. Aufl., 2. Band, S. $160 \mathrm{ff}$. 
schwächer wurde. Noch auffallender war die polsterförmige Verdickung der Zellwände in den Einbuchtungen zwischen den kurzen Armen der Schwammparenchymzellen (Fig. 4, 5). Die Membrandicke betrug hier bis zu $4.6 \mu$, während die Wandung der Arme gewöhnlich bloß 0.8 bis $0.9 \mu$ dick war. Die Verdickungen zeigten mit Chlorzinkjod stets die normale Cellulose-Reaction, ebenso die übrigen Wandtheile.

5. Der Turgordruck war in herangewachsenen Zellen der Culturen größer als in den normalen, im Gewebsverbande befindlichen Zellen. Da diese Zunahme des osmotischen Druckes sowohl bei der Cultur in Knop'scher Nährstofflösung, wie auch in 1 - bis 3 procentiger Zuckerlösung eintrat, so konnte es sich nicht um eine Anpassung an concentriertere Nährlösungen handeln, zumal die Zunahme des osmotischen Druckes im Verhältnisse zur Concentration der Lösungen eine sehr große war. Während in normalen Assimilationszellen zu Beginn derVersuche die Plasmolyse bereits in 3 procentiger Kalisalpeterlösung eintrat, stellte sie sich in den herangewachsenen Zellen zweier 14 tägiger Culturen in Nährsalzlösung und 1 procentiger Zuckerlösung erst nach Zusatz von 5 procentiger Kalisalpeterlösung ein. Das käme also einer Steigerung des Turgordruckes von $10 \cdot \overline{\text { auf }} 17 \cdot 5$ Atmosphären gleich. Auffallend war aber das baldige Zurückgehen der Plasmolyse in den cultivierten Zellen. Die Plasmahaut (Vacuolenwand) wurde also für Kalisalpeter bald durchlässig. Das deutet auf eine krankhafte Modificierung der Vacuolenwand hin. Sollte dieselbe schon zu Beginn der plasmolytischen Versuche eine gewisse Permeabilität für Kalisalpeter besessen haben, was mir ziemlich wahrscheinlich ist, so wäre der oben gefundene Wert für den Turgordruck natürlich zu hoch bemessen.

Nicht selten beobachtete ich bei Plasmolyse mit 5 procentiger Salpeterlösung oder mit verdünntem Glycerin bloß Abhebung und Contraction der Vacuolenwand, während die äußere Plasmahaut und das Körnerplasma sammt dem Zellkern und den Chloroplasten an der Zellwand haften blieben. Dieselbe Erscheinung constatierte $\mathrm{H}$. de Vries ${ }^{1}$ an Zellen, deren

1 H. de Vries, Plasmolytische Studien über die Wand der Vacuolen. Jahrb. f. wissensch. Bot., 16. Bd., S. 466, 467. 
Protoplasma in langsamem Absterben begriffen war. Für die Annahme, dass bei den betreffenden Zellen in meinen Culturen die genannten Plasmatheile bereits vor Beginn der Plasmolyse todt gewesen seien, liegt kein triftiger Grund vor. Dem Absterben des Plasmaschlauches gehen nämlich, wie gleich gezeigt werden wird, charakteristische Veränderungen voraus, die in den oben erwähnten Fällen noch nicht eingetreten waren. Wohl aber muss angenommen werden, dass das Cytoplasma mit Ausschluss der widerstandsfähigeren Vacuolenwand bereits so sehr geschwächt war, dass es durch die plötzliche Einwirkung der 5 procentigen Salpeterlösung rasch getödtet wurde. Auch de Vries weist darauf hin, dass Zellen, deren Protoplasma durch längeres Verweilen der Schnitte in Lösungen indifferenter Substanzen oder durch Zusatz äußerst geringer Mengen giftiger Substanzen oder durch langsames Erwärmen bis zur oberen Temperaturgrenze des Lebens geschädigt wird, nach plötzlicher Einwirkung der Salpeterlösung nur noch die Abhebung und Contraction der resistenteren Vacuolenwand erkennen lassen. In meinen Culturen war aber der Schädigung des Cytoplasmas ein kräftiges Wachsthum der Protoplasten vorausgegangen.

Bei diesen Versuchen wurden auch einige Messungen bezüglich der Dimensionsverhältnisse der Palissadenzellen vor und nach der Plasmolyse vorgenommen. Nachstehende kleine Tabelle enthält die gefundenen Werte in Theilstrichen des Ocularmikrometers:

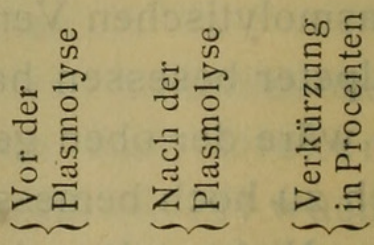

1. Palissadenzelle, frisch dem (Länge.. $20 \cdot 8 \quad 19 \quad 8 \cdot 6$ $\begin{array}{lllll}\text { Blatte entnommen } \quad \text { Breite } & . & 8 & 7 \cdot 5 & 6 \cdot 2\end{array}$

2. Palissadenzelle nach 10 tägiger $\left\{\right.$ Länge $\ldots \begin{array}{llll}37 \cdot 5 & 35 \cdot 8 & 4 \cdot 5\end{array}$

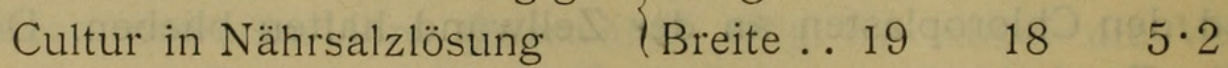

3. Palissadenzelle nach 10tägiger (Länge . $\begin{array}{llll}38 & 37 & 2 \cdot 6\end{array}$ Cultur in $1 \%$ Rohrzuckerlösung (Breite .. $18 \cdot 8 \quad 18 \quad 4 \cdot 2$ 
Es ergibt sich daraus, dass die Verkürzung des Längsdurchmessers der Zellen bei Aufhebung des Turgordruckes relativ bedeutend größer ist, wenn die Zellen frisch dem Blatte entnommen sind (Verkürzung $8 \cdot 6 \%$ ), als wenn sie aus einer 10 tägigen Cultur stammen (Verkürzung $4 \cdot 5$ und $2 \cdot 6 \%$ ). Viel geringer ist der Unterschied in der Verkürzung des Querdurchmessers. Ob diese geringere Dehnung der Zellwände cultivierter und stark gewachsener Palissadenzellen (trotz des stärkeren Turgordruckes) auf die Zunahme der Wanddicke zurückzuführen ist, oder ob die Dehnbarkeit selbst abgenommen hat, also eine qualitative Änderung in den mechanischen Eigenschaften der Membran eingetreten ist, muss dahingestellt bleiben.

6. Über Änderungen in der Beschaffenheit des Ze11kernes der cultivierten Zellen liegen mir bloß wenige Beobachtungen vor. In den normalen Assimilationszellen besitzt der halblinsenförmige, der Zellwand angelagerte Zellkern einen Durchmesser von circa $6 \mu$. In einer 5 tägigen Cultur (Knop'sche Nährstofflösung $+1 \%$ Rohrzucker) betrug der Kerndurchmesser 8 bis $10 \mu$. Mit den Zellen sind also auch die Kerne gewachsen. Dagegen waren in einer 16 tägigen Cultur (Knop'sche Nährstofflösung), in der die stark gewachsenen Zellen bereits im Absterben begriffen waren, die Kerne wieder beträchtlich kleiner. Ihr Durchmesser betrug jetzt nur noch 3 bis $4.5 \mu$.

7. Dem Absterben der Zellen gieng in allen Culturen eine unregelmäßige scharfe Fältelung des ganz dünn gewordenen Plasmaschlauches voraus. Der Plasmabeleg hob sich an verschiedenen Stellen von der Zellwand ab - in den Schwammparenchymzellen zunächst über den polsterförmigen Wandverdickungen in den Zellbuchten - und stülpte sich dann, schmale Falten bildend, oft ziemlich tief in das Zellumen ein. Diese Faltenbildung kann nicht bloß eine plasmolytische Erscheinung sein, denn sie ist mit einer entsprechenden Oberflächenvergrößerung des Plasmaschlauches verknüpft. Es muss vielmehr angenommen werden, dass sie auf activem Flächenwachsthum des Plasmaschlauches beruht, die zur Faltenbildung führt, weil die Zellwand die Fähigkeit zum Flächenwachsthum bereits eingebüßt hat. 
Später, beim Absterben, hebt sich allerdings der ganze Plasmabeleg von der Zellwand ein wenig ab (Fig. 5). Er zeigt jetzt in der Flächenansicht unregelmäßig gewundene, oft zickzackförmig gebrochene, scharfe Linien, die zum Theile sicher ganz schmalen, scharfen Falten entsprechen, zum Theile aber feine Risslinien vorstellen dürften.

\section{III.}

Ich will jetzt noch die Ergebnisse der mit den isolierten Zellen anderer Pflanzen durchgeführten Culturversuche mittheilen. Allerdings handelte es sich dabei bloß um vereinzelte Versuche, die aber immerhin einige bemerkenswerte Resultate ergaben.

Am 29. September wurden zwei Culturversuche in Schälchen mit den isolierten Assimilationszellen des Laubblattes von Eichhornia crassipes begonnen. In beiden Versuchen kam Knop'sche Nährstofflösung zur Verwendung. Die eine Cultur wurde am Fenster dem diffusen Tageslichte ausgesetzt, die andere wurde verdunkelt. Beide Culturen enthielten vollkommen stärkefreie Assimilationszellen vermischt mit solchen, deren Chlorophyllkörner reichlich Stärkeeinschlüsse aufwiesen.

In der belichteten Cultur waren nach 5 Tagen die stärkefreien Zellen fast sämmtlich noch am Leben; einige waren ziemlich stark gewachsen. Die Chlorophyllkörner sind auffallend kleiner und blasser geworden. Von den stärkeführenden Zellen ist eine größere Anzahl abgestorben; die Stärkemenge in den nur wenig verblassten Chlorophyllkörnern hat anscheinend nur wenig oder gar nicht abgenommen.

In der verdunkelten Cultur waren die stärkelosen Zellen nach 5 Tagen wenig oder gar nicht gewachsen; ihre zerstreuten Chlorophyllkörner sind auffallend kleiner geworden und von lichtgelber Farbe. In den stärkeführenden Zellen hat die Stärkemenge in den gleich groß und lebhaft grün gebliebenen Chlorophyllkörnern mehr minder abgenommen. Nach 10 Tagen zeigten stärkelose und stärkeführende Zellen im allgemeinen keine weitere Veränderung. In den stärkelosen waren die vergilbten kleinen Chlorophyllkörner oft rings um den Kern 
gelagert, in den stärkeführenden waren sie noch immer von gleicher Größe und schön grüner Farbe.

Dieser Versuch lehrt also, dass die Chlorophyllkörner isolierter Zellen im Dunkeln bald degenerieren, wenn sie zu Beginn des Versuches stärkefrei waren, während sie intact bleiben, wenn sie bei mangelndem oder geringfügigen Wachsthume der Zellen die in ihnen aufgespeicherte Stärke wenigstens theilweise für sich verwenden können.

Auch mit chlorophyllosen Zellen wurden Züchtungsversuche ausgeführt. An den Laubblättern von Pulmonaria mollissima Kern. kommen zahlreiche "Drüsenhaare "vor, deren keulenförmig gestreckte, oft schräg aufsitzende Endzellen von gleichmäßig körnigem Plasma vollständig erfüllt sind. Der central gelegene runde Kern besitzt ein großes Kernkörperchen (Fig. 11). Diese Drüsenhaare wurden mit dem Rasiermesser so abgeschnitten, dass mit den Drüsenzellen gewöhnlich auch noch ein oder zwei intacte Stielzellen in die Cultur gelangten.

In gewöhnlichem Leitungswasser, sowie in Knop'scher Nährstofflösung traten ungefähr gleichzeitig dieselben Veränderungen ein, doch blieben die Zellen in Leitungswasser etwas länger am Leben. Schon nach einem Tage traten im Plasma der Drüsenzellen zahlreiche, meist längsgestreckte Vacuolen auf, so dass dasselbe einen grobfaserigen Bau erhielt (Fig. 12). Die Plasmafasern waren alle parallel zur Längsaxe der Zelle angeordnet. In der Nähe des Zellkernes war das Plasma feinschaumig. Stark lichtbrechende Tröpfchen in spärlicher Anzahl waren unregelmäßig im Plasma vertheilt. Nach 3 Tagen waren die Drüsenzellen noch plasmaärmer geworden. Es hatte sich ein plasmatischer Wandbeleg ausgebildet, der durch eine Anzahl von Plasmabalken und -Fäden mit einer centralen Plasmapartie im Zusammenhange stand, welche den etwas kleiner gewordenen Kern enthielt (Fig. 13). Nach 7 Tagen waren die in der Nährstofflösung befindlichen Zellen sämmtlich abgestorben. In Leitungswasser waren noch einige am Leben. Ihr Plasmakörper war nun sehr stark reduciert und substanzarm (Fig. 14); er bildete nur mehr einen dünnen Wandbeleg, der an der Basis der Zelle über der Querwand 
etwas dicker war; hier trat nunmehr auch der auffallend klein gewordene Zellkern auf. Von der dünnen Plasmahülle, die ihn umgab, strahlten einige Plasmafäden gegen die benachbarten Wandpartien aus. Wachsthum der Zellen ließ sich in keinem Falle beobachten.

In einer dritten Cultur, die gleichzeitig durchgeführt wurde, befanden sich die Drüsenzellen in einer Nährlösung, die $3 \%$ Rohrzucker und $1 \%$ Asparagin enthielt. Nach einem Tage waren die Zellen noch kaum verändert; nur einzelne zeigten Andeutungen jener faserigen Differenzierung des Plasmas, die in den früher besprochenen Culturen schon nach dem ersten Tage so deutlich zu beobachten war. Nach 3 Tagen zeigte das Plasma dieselbe Vertheilung, wie bei der Cultur in Leitungswasser, doch war es noch reichlicher vorhanden und stark körnig. Am 7. Tage waren zahlreiche Zellen todt, in den noch lebenden zeigte der Protoplast wieder dasselbe Bild, wie in Wasser, doch war er kräftiger, substanzreicher. Auch in dieser Cultur stellte sich kein Wachsthum der Zellen ein.

Die Abmagerung des anfänglich so kräftig entwickelten Protoplasten der Drüsenzellen bei der Cultur in Leitungswasser und Nährsalzlösung war vorauszusehen, da sich die Zellen im Hungerzustande befanden. Überraschend ist dabei einigermaßen die Schnelligkeit, mit der der Substanzverlust erfolgte. Da nicht anzunehmen ist, dass die Plasmahaut nennenswerte Mengen plastischer Baustoffe durch sich austreten ließ, so müssen sich in den Protoplasten sehr lebhafte Stoffwechselprocesse abgespielt haben, deren Endproducte ausgeschieden wurden. Vor allem dürfte die Athmung eine sehr lebhafte gewesen sein. Dass in den Plasmakörpern der Drüsenzellen der Stoffwechsel ein besonders reger ist, muss ja auch aus anderen Gründen angenommen werden. Ernährung mit organischen Stoffen (Rohrzucker und Asparagin) verlangsamte zwar die Abmagerung der Protoplasten, war aber bei der angegebenen Concentration der Nährlösungen nicht imstande, sie hintanzuhalten.

Ein eigenthümliches Verhalten zeigten abgeschnittene Brennhaare von Urtica dioica, die in Nährsalzlösung, der $1 \%$ Rohrzucker zugesetzt war, im Thermostaten bei einer 
Temperatur von $33^{\circ} \mathrm{C}$. gezüchtet wurden. Beginn des Versuches am 22. September. Nach 2 Tagen waren noch alle Brennhaarzellen am Leben. Nach 10 Tagen war die Mehrzahl abgestorben; die am Leben gebliebenen zeigten folgendes Aussehen: Der sonst plasmareiche Bulbus der Brennhaarzelle besaß jetzt nur einen relativ dünnen plasmatischen Wandbeleg und einen dicken, längsfaserigen, centralen Plasmastrang. Der Kern war aus dem Bulbus hinausgerückt und befand sich nun in der Basis des kegelförmigen Theiles des Haarkörpers. In diesem war nun sehr reichlich Plasma vorhanden; an Stelle des einheitlichen Saftraumes traten jetzt zahlreiche, verschieden große, ellipsoidisch gestreckte Vacuolen auf. Der obere Theil des Haares war ganz von Plasma erfüllt, ohne Vacuolen. Das Plasma zeigte langsame Strömung und besa $\beta$ in sehr ausgeprägtem Maße jene längsfaserige Structur, die strömende Plasmamassen so häufig erkennen lassen. ${ }^{1} \mathrm{Am}$ 7. October, d. i. nach 15 Tagen, war alles todt und stark verpilzt.

Bemerkenswert war bei diesem Versuche erstens die Umlagerung des Protoplasmas, von dem ein großer Theil aus dem Bulbus in die obere Haarpartie auswanderte, und zweitens die Thatsache, dass die Gesammtmenge des Protoplasmas nach 10 Tagen noch nicht abgenommen, sondern, soweit die Schätzung beurtheilen ließ, eher zugenommen hatte. Es ist nicht unmöglich, dass die Regenerierung und das Wachsthum des Plasmakörpers auf Kosten der zugeführten Nährstoffe erfolgt ist. Wahrscheinlicher ist mir aber, dass die beträchtlichen Eiweißmengen, die im Zellsafte des Brennhaares gelöst sind, ${ }^{2}$ einen Reservestoff vorstellten, der die Abmagerung des Protoplasten verhinderte, ja eventuell sogar sein weiteres Wachsthum ermöglichte.

Ein noch interessanteres Ergebnis hatte ein Culturversuch mit den Zellen der Staubfadenhare von Tradescantia

1 Vergl. G. Haberlandt, Über fibrilläre Plasmastructuren. Berichte der deutschen bot. Gesellschaft, 1901, Decemberheft.

2 Vergl. G. Haberlandt, Zur Anatomie und Physiologie der pflanzlichen Brennhaare. Diese Sitzungsberichte, 93. Bd., I. Abth., 1886. 
virginica. Die noch nicht ganz ausgewachsenen Haare wurden zerschnitten und in vier-bis achtzelligen Fragmenten in einen hängenden Tropfen der Nährlösung gebracht, die $2 \%$ Traubenzucker und $0.4 \%$ Asparagin enthielt. Gewöhnlich starben in den Theilstücken alle Zellen bis auf 1 bis 2 ab, so dass thatsächlich einzelne Zellen gezüchtet wurden. Überraschend war nun vor allem die lange Lebensdauer dieser isolierten Haarzellen. Noch nach 26 Tagen waren zahlreiche Zellen am Leben. Durch die künstliche Ernährung konnte also ihre Lebensdauer weit über das Normale hinaus verlängert werden. Dabei zeigten die Zellen ein sehr kräftiges Wachsthum, sie nahmen in Länge und Breite ungefähr um das Doppelte zu und wiesen auch kräftig entwickelte Plasmakörper auf (Fig. 7). Die Vertheilung des Plasmas war die normale.

Sehr auffallend war das Verhalten der an todte Nachbarzellen grenzenden Querwände. Dieselben wurden natürlich infolge des Turgordruckes in die Lumina der abgestorbenen Nachbarzellen vorgewölbt und zeigten meist ein ganz auffälliges Dickenwachsthum (Fig. 8 bis 10). Die Membran wurde schließlich 3 - bis 5 mal so dick, als sie anfänglich war. Häufig erstreckte sich die Verdickung auch auf die angrenzenden Partien der Außenwände und verlor sich hier allmählich. Bemerkenswert war auch die Lage des Zellkernes. Wenn die betreffende Zelle beiderseits an todte Zellen grenzte und so ihre beiden papillös vorgestülpten Querwände verdickte, lag der Zellkern annähernd in der Mitte. Wenn aber zwei benachbarte Zellen am Leben blieben (Fig. 7), zwischen denen die Scheidewand eben und unverdickt blieb, dann rückten die beiden Kerne bis knapp an die sich verdickenden Querwände heran: ein weiteres instructives Beispiel für die von mir vor Jahren geschilderten Beziehungen zwischen Function und Lage des Zellkernes in wachsenden Pflanzenzellen.

Dass nach Verletzung eines mehrzelligen Haares die Querwand, welche nunmehr zur Außenwand wird, sich verdickt und cutinisiert, kommt häufig vor und ist eine leicht verständliche biologische Schutzeinrichtung. Bei Haaren, die im Zusammenhange mit der Gesammtpflanze stehen, ist es aber fraglich, ob die Verdickung und Cutinisierung der 
Querwand eine ausschließliche Reaction der betreffenden Haarzelle ist, oder ob diese von dem ganzen Organe gewissermaßen den Auftrag erhält, ihre bloßgelegte Außenwand zu verdicken. Das Ergebnis unseres Culturversuches mit Haarfragmenten von Tradescantia lehrt, dass ersteres zutrifft. Die betreffende Zelle verdickt ihre Querwand aus eigener Initiative und bekundet derart auch den Selbsterhaltungstrieb des Elementarorganismus.

Gewöhnliche Epidermiszellen lassen sich, soweit meine allerdings sehr lückenhaften Beobachtungen reichen, im isolierten Zustande nur eine kurze Zeit lang am Leben erhalten. An kleinen, abgezogenen Epidermisstückchen des Laubblattes von Ornithogalum fiel mir auf, dass die zwischen den langen Epidermiszellen eingeschalteten Kurzzellen einige Tage länger am Leben blieben, als die ersteren.

Auf die große Lebenszähigkeit der Spaltöffnungszellen hat bereits Leitge ${ }^{1}$ hingewiesen. Er ließ z. B. $1 \mathrm{~cm}$ lange Schaftstücke von Galtonia candicans einen Monat lang im feuchten Raume (am Lichte) stehen. Sie waren nach dieser Zeit missfarbig geworden, verfault und von Pilzen durchwachsen. Alle Gewebe waren abgestorben, nur die Schließzellen waren lebhaft grün, stark turgescierend und außerordentlich stark gekrümmt, so dass die einzelne Schliesszelle sich mit ihren Enden berührte und einen förmlichen Ring bildete. Nach Aufhebung des Turgors trat kein vollständiger Spaltenverschluss mehr ein, was Leitgeb auf die enorm starke, die Elasticitätsgrenze überschreitende Dehnung der Zellwände zurückführt. Vielleicht war aber auch schon Flächenwachsthum der Membranen, speciell der Rückenwände, eingetreten. - Auch ich beobachtete in meinen Culturen die von Leitgeb beschriebenen Erscheinungen, und zwar bei verschiedenen Pflanzen (Ornithogalum umbellatum, Erythronium dens canis, Fuchsia globosa), kann aber seinen Angaben nichts wesentlich Neues hinzufügen. Jedenfalls empfehlen sich die Schließzellen wegen ihrer großen Widerstandsfähigkeit sehr für derartige Culturversuche.

1 Beiträge zur Physiologie der Spaltöffnungsapparate. Mittheilungen aus dem bot. Institute zu Graz, 2. Heft, 1888, S. 123. 


\section{IV.}

Zum Schlusse möchte ich noch auf den bemerkenswerten Umstand hinweisen, dass in meinen Zellculturen trotz des so auffallenden Wachsthums der Zellen, das sich häufig einstellte, niemals Zelltheilungen $z u$ beobachten waren. Es wird nun Aufgabe künftiger Culturversuche sein, die Bedingungen ausfindig zu machen, unter denen isolierte Zellen zur Theilung schreiten. Gewisse Fingerzeige geben in dieser Hinsicht die bekannten Versuche von Loeb, Nathansohn und Hans Winkler über die experimentell herbeigeführte Weiterentwickelung, respective Furchung unbefruchteter Eizellen.

Loeb ${ }^{1}$ hat zuerst an Eiern von Seeigeln, die nach einbis zweistündigem Verweilen in einer $\mathrm{MgCl}_{2}$-Lösung in gewöhnliches Seewasser zurückgebracht wurden, eine parthenogenetische Weiterentwickelung beobachtet und daraus auf eine specifische Wirkung des $\mathrm{Mg}$ geschlossen. Spätere Versuche lehrten ihn aber, dass geeignete Concentrationen von $\mathrm{NaCl}$ und $\mathrm{KCl}$, sowie von Rohrzucker die gleiche Wirkung haben, so dass nur eine bestimmte Erhöhung des osmotischen Druckes der die Eier umgebenden Flüssigkeit die parthenogenetische Weiterentwickelung bedingen konnte. Es wird nun $\mathrm{zu}$ untersuchen sein, ob vielleicht derartige Versuche mit isolierten Pflanzenzellen zu einer Theilung derselben führen.

Von Nathansohn ${ }^{2}$ wurde gezeigt, dass die bei der Gattung Marsilia vorhandene Tendenz zur parthenogenetischen Entwickelung der Eizellen durch Temperaturerhöhung (auf $35^{\circ}$ ) gesteigert werden kann.

Nachdem ich die Mittheilung Nathansohn's gelesen hatte, führte ich noch einige nachträgliche Culturversuche mit isolierten Zellen bei einer Temperatur von $33^{\circ}$ C. (im

1 J. Loeb, On the nature of the process of fertilization etc. Americ. Journ. of physiology, Bd. III, 1899. Ferner: Experim. on artifical parthenogenesis in Auclids (Chaetopterus) and the nature of fertilization. Ebenda, Bd. IV, 1901.

2 Über Parthenogenesis bei Marsilia und ihre Abhängigkeit von der Temperatur. Berichte der Deutschen bot. Gesellschaft, 1901, S. $99 \mathrm{ff}$. 
Thermostaten) aus. Die Assimilationszellen zeigten dabei kein anderes Verhalten, als bei gewöhnlicher Zimmertemperatur, nur traten die beschriebenen Veränderungen rascher ein und dementsprechend giengen die Zellen auch früher zugrunde. Das Ergebnis des Culturversuches mit Brennhaaren von Urtica dioica wurde schon oben beschrieben.

Zuletzt ist von Hans Winkler ${ }^{1}$ der Nachweis erbracht worden, dass unbefruchtete Eier von Arbacia und Sphaerechinus durch Extractivstoffe aus dem Sperma derselben Species zu einigen Theilungen veranlasst werden. Dieses Ergebnis findet in gewisser Hinsicht sein Analogon in der auch von Winkler erwähnten anregenden Wirkung des Pollenschlauches auf die Ausbildung der Samenknospen bei Orchideen, auf die Anschwellung des Fruchtknotens etc. Wahrscheinlich handelt es sich auch hier um Substanzen — »Wuchsenzyme « — die, aus dem Pollenschlauche austretend, durch stoffliche Reizung die betreffenden Zellen zum Wachsthum und zur Theilung anregen. Natürlich müssen diese Substanzen nicht identisch sein mit jenen, die eventuell die Theilung der Eizelle bewirken, wie ja überhaupt die zur Theilung der Eizelle führenden Reize verschieden sein können von jenen, die vegetative Zellen zur Theilung veranlassen. ${ }^{2}$ Immerhin würde es sich verlohnen, im hängenden Tropfen gleichzeitig isolierte vegetative Zellen und Pollenschläuche zu cultivieren; vielleicht regen die letzteren jene zur Theilung an. Am aussichtsvollsten wären derartige Versuche mit Orchideen und anderen Pflanzen, bei denen die oben erwähnte Wirkung der Pollenschläuche bereits sicher festgestellt ist.

Allein nicht bloß Pollenschläuche wären heranzuziehen, um vegetative Zellen zur Theilung zu zwingen. Man könnte den betreffenden Nährlösungen auch Extracte aus Vegetationsspitzen zusetzen, beziehungsweise die Zellen in solchen

1 Über die Furchung unbefruchteter Eier unter der Einwirkung von Extractivstoffen aus dem Sperma. Nachricht der k. Gesellsch. der Wissensch. zu Göttingen, Math.-phys. C1., 1900, Heft 2. Ferner: Über Merogonie und Befruchtung. Jahrb. für wissensch. Botanik, 36. Bd., 1901.

2 Vergl. Winkler, Jahrb. für wissensch. Botanik, 36. Bd., S. 773. 
cultivieren. Auch an die Verwendung von Embryosacksäften wäre zu denken.

Ohne mich auf weitere Fragestellungen einzulassen, glaube ich zum Schlusse keine allzu kühne Prophezeiung auszusprechen, wenn ich auf die Möglichkeit hinweise, dass es auf diese Weise vielleicht gelingen wird, aus vegetativen Zellen künstliche Embryonen zu züchten.

Jedenfalls dürfte die Methode der Züchtung isolierter Pflanzenzellen in Nährlösungen verschiedene wichtige Probleme von einer neuen Seite her der experimentellen Bearbeitung zugänglich machen. 


\section{Erklärung der Abbildungen.}

Fig. 1. Palissadenzelle von Lamium purpureum nach 8 tägiger Cultur in Nährsalzlösung, stark gewachsen. Unten eine bei der Isolierung verletzte und abgestorbene Palissadenzelle.

Fig. 2. Zwei Palissadenzellen von Lamium purpureum nach 8 tägiger Cultur in Nährsalzlösung, stark gewachsen.

Fig. 3. Theil einer Palissadenzelle von Lamium purpureum nach 11 tägiger Cultur in 1procentiger Rohrzuckerlösung; Plasmolyse durch verdünntes Glycerin. Die obere Querwand hat sich verdickt.

Fig. 4. Schwammparenchymzelle von Lamium purpureum nach 12 tägiger Cultur in Nährsalzlösung; polsterförmige Membranverdickungen in den Zellbuchten; der Zellinhalt ist nicht eingetragen.

Fig. 5. Abgestorbene Schwammparenchymzelle von Lamium purpureum nach 16 tägiger Cultur in Nährsalzlösung; der abgehobene Plasmaschlauch zeigt Fältelung.

Fig. 6. Chlorophyllkörner der Palissadenzellen von Lamium purpureum nach 5 tägiger Cultur in 5 procentiger Rohrzuckerlösung; Cultur im Dunkeln.

Fig. 7. Stück eines Staubfadenhaares von Tradescantia virginica nach 12 tägiger Cultur in Nährstofflösung ( $2 \%$ Traubenzucker, $0.4 \%$ Asparagin). Nur zwei Zellen sind am Leben geblieben; dieselben sind gewachsen und haben ihre an die todten Zellen angrenzenden Querwände verdickt.

Fig. 8. Ende einer lebenden Haarzelle nach 8 tägiger Cultur; die Querwand hat sich papillös vorgestülpt und stark verdickt.

Fig. 9 und 10. Desgleichen nach 12 tägiger Cultur. Die Membranverdickung erstreckt sich auch über die an die Querwand angrenzenden Partien der Außenwand.

Fig. 11. Endzelle eines Drüsenhaares von Pulmonaria mollissima Kern. zu Beginn des Culturversuches.

Fig. 12. Desgleichen nach eintägiger Cultur in Leitungswasser. Vacuolisierung des Plasmas, das theilweise eine grob längsfaserige Structur annimmt.

Fig. 13. Desgleichen nach 3 tägiger Cultur in Leitungswasser.

Fig. 14. Desgleichen nach 7 tägiger Cultur in Leitungswasser. Der Protoplast ist äußerst abgemagert, der Zellkern viel kleiner geworden. 


\section{$2 \mathrm{BHL}$ Biodiversity Heritage Library}

Haberlandt, Gottlieb. 1902. "Culturversuche mit isolierten Pflanzenzellen." Sitzungsberichte 111, 69-91.

View This Item Online: https://www.biodiversitylibrary.org/item/110031

Permalink: https://www.biodiversitylibrary.org/partpdf/233868

\section{Holding Institution}

Smithsonian Libraries

\section{Sponsored by}

Biodiversity Heritage Library

\section{Copyright \& Reuse}

Copyright Status: Public domain. The BHL considers that this work is no longer under copyright protection.

This document was created from content at the Biodiversity Heritage Library, the world's largest open access digital library for biodiversity literature and archives. Visit BHL at https://www.biodiversitylibrary.org. 